\title{
GENTAMICIN BIOAUTOGRAPHY ASSAY VS. THE MICROBIOLOGICAL DISK TEST
}

\author{
Arie Rosner and Haim Aviv \\ Department of Virology, Weizmann Institute of Science, Rehovot, Israel \\ (Received for publication January 9, 1980)
}

\begin{abstract}
The use of bioautography for quantitative measurement of gentamicin concentrations was compared with the disk test. Following chromatographic separation and bioautography, gentamicin produced inhibition zones, $2 \sim 7$ times larger than the inhibition zones formed by the same amounts of gentamicin in the disk test. Bioautography, therefore, is a more sensitive assay method.
\end{abstract}

Gentamicin is a broad spectrum antibiotic complex of the aminoglycoside group ${ }^{1)}$ which consists of several components: $\mathrm{C}_{1}, \mathrm{C}_{1 \mathrm{a}}$ and $\mathrm{C}_{2}$ when analyzed by bioautography ${ }^{2}$. It is active against both Gram-negative and Gram-positive bacteria. However, therapeutic treatment requires monitoring of gentamicin blood levels, to control high serum concentrations that may have adverse side-effects ${ }^{3)}$. The assays for gentamicin commonly used are the microbiological $1^{42}$ and enzymatic methods ${ }^{52}$. Recently, spectrophotometric ${ }^{(1)}$, radioimmunoassay ${ }^{7,8)}$, high pressure liquid chromatography ${ }^{9)}$ and gasliquid chromatography ${ }^{10)}$ methods have also been introduced.

Gentamicin is produced by the genus Micromonospor ${ }^{11)}$. Its measurement in growth medium extracts by the disk test was found to be variable, due to interference by substances contained in the medium. Therefore bioautography was applied. The results of measurements of gentamicin by these two methods were compared.

\section{Materials and Methods}

\section{Gentamicin fermentation}

Micromonospora purpurea ATCC 15835 was grown in $100 \mathrm{ml}$ of medium $\mathrm{S}^{12)}$ for $4 \sim 6$ days. Gentamicin was purified from the medium on IRC-50 columns as previously detailed by LEE et al. ${ }^{13)}$

Microbiological disk test

All gentamicin samples were made up to $30 \mu \mathrm{l}$ with water and applied to $0.8 \times 6 \mathrm{~mm}$ filter paper disks (Bel-Gar Ltd.). The filters were dried at $37^{\circ} \mathrm{C}$ and placed in Petri dish on a soft agar top layer seeded with Bacillus subtilis ATCC 6633. The soft agar contained $0.5 \%$ yeast extract (Difco), $1 \%$ Tryptone (Difco), $0.5 \% \mathrm{NaCl}$ and $0.75 \%$ Bactoagar (Difco). The Petri dishes were incubated overnight at $30^{\circ} \mathrm{C}$. The diameter of the inhibition zones formed around the filters were determined and the area of inhibition calculated.

\section{Bioautography}

A chloroform - methanol - ammonium hydroxide (2:1:1) solvent system was used in descending paper chromatography ${ }^{2)}$ of gentamicin on a Whatmann No. 1 paper. The microbiological detection of the gentamicin spots on the paper was performed, using Bacillus subtilis ATCC 6633 as indicator $\operatorname{organism}^{2)}$. The total area of inhibition was determined by drawing the contour of the gentamicin inhibition spots on a paper which was cut and weighed. The actual area of inhibition was calculated from the weight of the paper. 


\section{Results}

Applying the disk test for measuring gentamicin in Micromonospora medium extracts, we have sometimes observed in control samples an inhibition zone formed in the absence of gentamicin (not shown). Looking for a more specific method, bioautography was applied and a gentamicin inhibition zone standard curve was constructed. Various amounts of gentamicin were separated by paper chromatography and bioautographed (Fig. $1 \mathrm{~A}$ ). The three characteristic gentamicin spots $\mathrm{C}_{1}, \mathrm{C}_{1 \mathrm{a}}$ and $\mathrm{C}_{2}{ }^{2)}$ were clearly detected. The total areas of inhibition in this bioautograph were calculated and plotted against the corresponding gentamicin concentrations (Fig. 2A). For comparison, a disk test was carried out in parallel for the same range of gentamicin concentrations (Figs. 1B and 2A). The ratio between the inhibition zone size in bioautography and in the disk test (Fig. 2B) was shown to increase from about 2 for a gentamicin concentration of $0.4 \mu \mathrm{g}$ to more than 7 at higher concentrations. This means that the inhibition zone produced by a given amount of gentamicin in bioautography is larger than that formed in

Fig. 1. Determination of gentamicin by bioautography and the disk test.

Bioautography (A) and the disk test (B) were carried out as described in Materials and Methods. One $\mu 1$ of Micromonospora medium extract (mic.) was used for both the disk test and the bioautography.

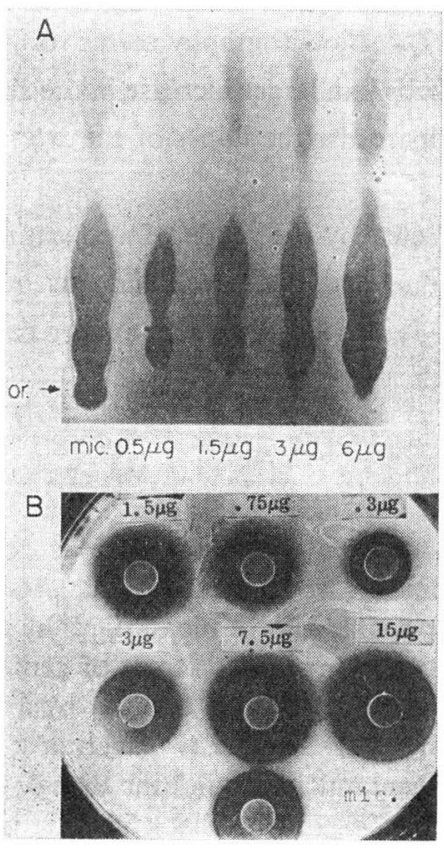

Fig. 2. Construction of gentamicin standard curves for bioautography and for the disk test.

(A) The experiment was similar to that described in Fig. $1 \mathrm{~A}$ and $\mathrm{B}$. The inhibition zone areas were calculated and plotted against the corresponding gentamicin concentrations, as described in Materials and Methods. The amount of gentamicin in the Micromonospora medium sample was determined by measuring the inhibition zone formed and by interpolation from the standard curves (open circles and interrupted lines).

(B) The ratio of the inhibition zone areas in bioautography $v s$. in the disk test was calculated from the data in (A) and plotted against the corresponding gentamicin concentration.

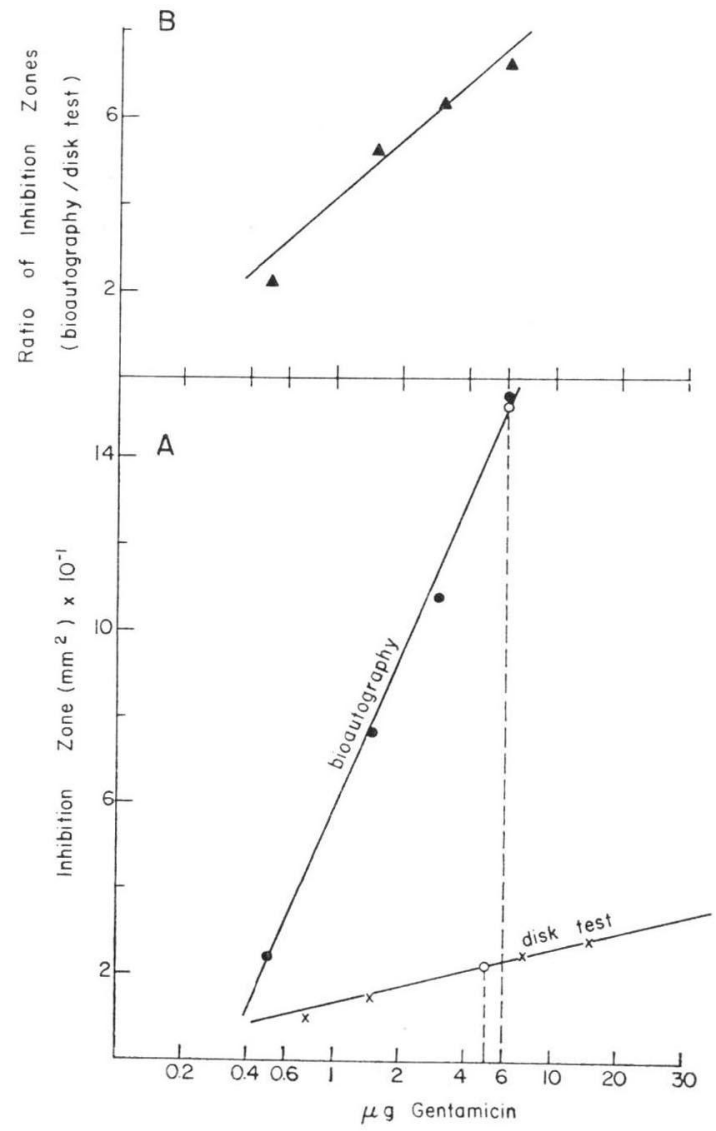


Fig. 3. The slopes of gentamicin standard curves in bioautography and in the disk test.

Several gentamicin standard curves were constructed independently in bioautography (open symbols), and the disk test (closed symbols), as detailed in the legends to Figs. 1 and 2. The slopes were calculated from these curves as the change in the inhibition zone area $\left(\mathrm{mm}^{2}\right)$ per $\mu \mathrm{g}$ of gentamicin.

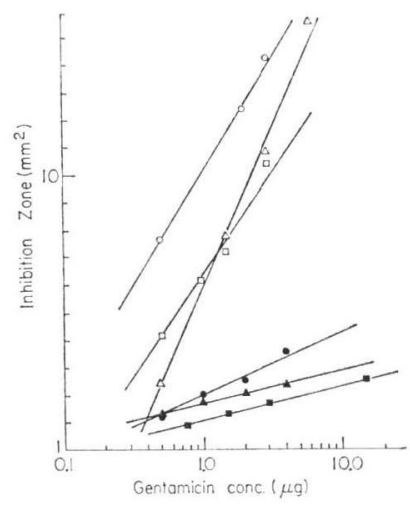

Fig. 4. The effect of sample volume on the size of the inhibition zone in bioautography.

Four samples containing $0.5 \mu \mathrm{g}$ of gentamicin were diluted in final volumes of $1,2,4$ and $6 \mu \mathrm{l}$ with water and applied onto chromatographic paper. Bioautography was carried out as detailed in Materials and Methods and the areas of inhibition were plotted against the diameters of the corresponding spots at the points of application, as measured before start of chromatography.

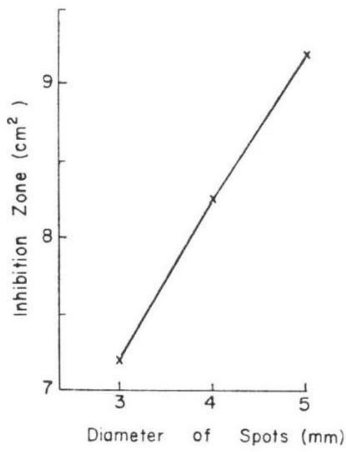

the microbiological diffusion disk test.

Using bioautography, (Fig. 2A), the gentamicin concentration in the sample of Micromonospora medium extract was measured to be $5.9 \mu \mathrm{g}$. In the disk test, in cases where minimum interference of medium was observed, similar value $(\sim 5 \mu \mathrm{g})$ was obtained.

In order to compare the slope of the inhibition zone curve in bioautography with that of the disk test, several independent gentamicin inhibition zone standard curves were constructed with each method and their slopes calculated (Fig. 3). The $\beta$ values of these slopes in bioautography (range $2.3 \sim 3.5$ ) are about six times higher than those of the disk test $(0.3 \sim 0.7)$. Bioautography seems to be a more desirable technique as a change in gentamicin concentration results in a larger increase in the size of the area of inhibition in bioautography than in the disk test. (Compare also the slopes of the two standard curves in Fig. 2A).

The size of the inhibition zone in bioautography was affected by the mode of application of the samples for the chromatography (Fig. 4); when the sample volume and, hence, the diameter of the spot at the point of application increased, the size of the inhibition zone also increased. Standard conditions, therefore, should be carefully maintained.

\section{Discussion}

Bioautography of gentamicin was previously used for the analysis of the various gentamicin components $^{2,14}$. Taking advantage of its high degree of specificity, we applied it in our present work as a quantitative tool. A standard curve was constructed for gentamicin in bioautography (Figs. 1A and $2 \mathrm{~A}$ ), and it was shown that the area of inhibition produced by a given amount of gentamicin in bioautography, was larger than that formed around filter disks with the same amount by a factor of $2 \sim 7$ (Fig. 2B). This is probably due to an essential difference between the two methods; while in the disk test the inhibition zone is created by the diffusion of gentamicin around the disk (which may release only a small fraction of the antibiotic, as most of it remains on the disk), in bioautography, 
in addition to its complete diffusion, the antibiotic is spread over a larger area during chromatography. Therefore it seems, that, due to the initial step of chromatographic separation and purification, bioautography is a more sensitive, as well as a highly specific method.

\section{Acknowledgements}

We thank Raya Zwang for excellent technical assistance and Ira Presser for valuable discussions.

\section{References}

1) Weinsteinn, M. J.; G. M. Luedemann, E. M. Oden \& G. H. Wagman: Gentamicin, a new broad-spectrum antibiotic complex. Antimicr. Agents \& Chemoth.-1963: 1 7, 1964

2) Wagman, G. H.; J. A. Marquez \& M. J. Weinstein: Chromatographic separation of the components of the gentamicin complex. J. Chromatogr. 34: 210 215, 1967

3) MAHON, W. A.; J. Ezer \& T. W. WiLson: Radioimmunoassay for measurement of gentamicin in blood. Antimicr. Agents \& Chemoth. 3: 385 389, 1973

4) Oden, E. M.; H. Stander \& M. J. Weinstein: Microbiological assay of gentamicin. Antimicr. Agents \& Chemoth.-1963: 8 13, 1964

5) Krooden, E. T. \& J. H. Darrell: Rapid gentamicin assay by enzymatic adenylation. J. Clinic. Pathol. 27: $452 \sim 456,1974$

6) Williams, J. W.; J.S. LANGer \& D. B. Northrop: A spectrophotometric assay for gentamicin. J. Antibiotics 28: 982 987, 1975

7) Berk, L. S.; J. L. Lewis \& J. C. Nelson: One hour radioimmunoassy of serum drug concentrations as exemplified by digozin and gentamicin. Clin. Chem. 20: 1159 1164, 1973

8) Broughton, A. \& J. E. Strong: Radioimmunoassay of iodinated gentamicin. Clinica Chimica Acta 66: $125 \sim 129,1976$

9) ANḦALt, J. P.: Assay of gentamicin in serum by high-pressure liquid chromatography. Antimicr. Agents \& Chemoth. 11: 651 655, 1977

10) MaYhew, J. W. \& S. L. Gorbach: Assay of gentamicin and tobramicin in sera of patients by gas-liquid chromatography. Antimicr. Agents \& Chemoth. 14: 851 855, 1978

11) Luedemann, G. M. \& B. C. Brodsky: Taxonomy of gentamicin-producing Micromonospora. Antimicr. Agents \& Chemoth.-1963: 116 124, 1964

12) Hopwood, D. A.; H. M. Wright, M. J. BibB \& S. N. Cohen: Genetic recombination through protoplast fusion in Streptomyces. Nature 268: 171 174, 1977

13) Lee, B. K.; R. G. Condon, G. H. Wagman, K. Byrne \& C. Schaffner: Incorporation of L-methioninemethyl ${ }^{14} \mathrm{C}$ into gentamicins. II. Large-scale preparation of methyl- ${ }^{14} \mathrm{C}$-gentamicins. J. Antibiotics 27: $822 \sim 825,1974$

14) Lee, B. K.; R. G. Condon, A. Murawski \& G. H. WaGman: Incorporation of L-methionine-methyl- ${ }^{14} \mathrm{C}$ into gentamicins. III. Chromatographic separation and degradation of components of methyl ${ }^{14} \mathrm{C}$-gentamicin complex. J. Antibiotics 28: 163 166, 1975 